\title{
Integration of PDMS and PMMA for Batch Fabrication of Microfluidic Devices
}

\author{
Hsih Yin Tan ${ }^{1}$, Weng Keong Loke ${ }^{1}$ and Nam-Trung Nguyen ${ }^{2}$ \\ ${ }^{1}$ DSO National Laboratories, Singapore, Singapore \\ ${ }^{2}$ School of Mechanical and Aerospace Engineering, Nanyang Technological University, Singapore, Singapore
}

\begin{abstract}
Polydimethylsiloxane (PDMS) finds its use in a wide range of microfluidic applications due to its flexibility and low cost . Poly(methyl methacrylate) (PMMA) attracts growing interest in microfluidics research community due to its low cost, high transparency, good mechanical and chemical properties. Combining PMMA with PDMS allows a high degree of flexibility in designing microfluidic devices. Bonding of PMMA to PDMS is a critical step for this hybrid approach. We present here a novel technique for bonding PMMA to PDMS. This technique is simple, fast and reliable. A dry adhesive layer was laminated onto the PMMA substrate. A precured PDMS mixture was spin coated onto the adhesive to form a PDMS membrane. The bonding quality and the strength of the PDMS/adhesive membrane was tested using a precision pressure source. The results show that the bond can hold a pressure up to $20 \mathrm{kPa}$.
\end{abstract}

Keywords - PDMS, PMMA, microfluidics, micropump, microvalve

\section{INTRODUCTION}

Since its emergence in the 1980s, microfluidics has been widely used in the development of systems such as microreactors for chemical, biological processes and lab-on-a-chip (LOC) for chemical and biochemical analysis [1]. Many LOC devices with microfluidic components for transport and mixing were fabricated in polymers [2,3]. Polymers were the main materials for realizing droplet-based microfluidics, a recent trend for sample preparation, transportation, reaction and detection within a droplet [4-6]. The recent rapid expansion of the biochip market leads to the need of cost effective materials and fabrication techniques. Polymers have become one of the most favoured materials for microfluidic devices, as they possess excellent optical properties, are disposable, biocompatible, easy and costeffective to fabricate and suitable for mass fabrication. A variety of polymeric materials are available with each having unique physical and chemical characteristics, thus making them suitable for different microfluidic applications. Some of these materials are thermoplastic polymers such as PMMA, polycarbonate (PC), cyclic olefin copolymer (COC) or cyclic olefin polymer (COP) and elastomers such as PDMS. PMMA and PDMS are the two most popular materials in the fabrication of microfluidic devices.
PDMS-based microfluidic devices form a class of components that are necessary for the realization of a fully integrated microfluidic system. A flexible PDMS membrane is an important component of a peristaltic micropump. Unger et. al. reported a multi-layer soft-lithography process for microfluidic systems [7]. Zhang et. al. [8] also reported a multi-layer micropump consisting of a thin layer of PDMS sandwiched between two PMMA layers. The PDMS acts as the actuating membrane and the PMMA layers accommodate both air chambers and micro ${ }^{\circ}$ uidic channels. The PDMS/PMMA bond is not strong and reversible. Kim et. al. modified PDMS with polypyrrole, a conductive polymer, to create a membrane that was encased in a PMMA structure [9].

PMMA is another popular polymeric material used in fabrication of microfuidic and nanofluidic [10] devices. This thermoplastic is cheap, easy to mass produce, rigid, transparent, impermeable to air, and compatible for electrokinetics. A number of machining techniques for PMMA devices such as hot embossing [11], injection moulding, laser ablation, and solvent imprinting have been reported. PMMA is too rigid to be used as membrane material in micropumps, but it is an excellent material for the air chambers. Numerous methods have been investigated and reported to bond polymeric substrates to obtain sealed microfluidic devices. As mentioned above, PDMS is a flexible material. Hence, there had been an increased interest to develop techniques to bond acylate materials to PDMS to make it more robust. Due to the different surface and chemical properties of these two materials, bonding of PDMS to PMMA is not an easy task. It was not possible to treat PDMS-PMMA bonding 4 both surfaces with oxygen plasma to initiate irreversible bond. There are a number of technologies reported for bonding of PDMS to PMMA, notable by CVD process, surface modi cation by silane/silicate coatings and adhesives [12, 13,14]. One of the methods of bonding PMMA to PDMS was modifying the mixture ratio of the PDMS elastomer and curing agent, followed by thermal bonding. Toh et. al. [12] spin coated the PDMS mixture onto a double-sided adhesive layer. This membrane was than sandwiched between two PMMA layers. A few papers reported modifying the PMMA surface using silanization. This method gives the modified PMMA surface a silane matrix similar to glass, allowing it to undergo plasma bonding to PDMS. Vlachopoulou et. al. [13] reported a method 


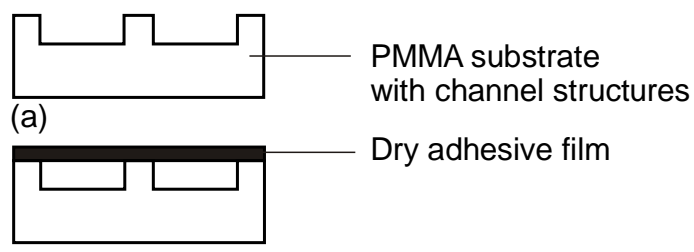

(b)

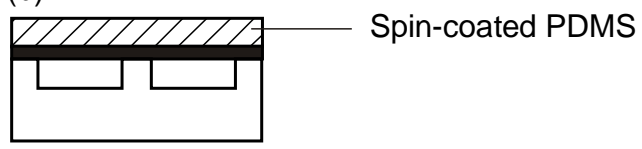

(c)

Fig. 2 Fabrication process of the PMMA/adhesive/PDMS part and the peristaltic micropupm: (a) PMMA wafer with engraved air chambers; (b) Dry adhesive - $\mathrm{lm}$ is laminated onto the PMMA air chambers; (c) PDMS is spin coated onto the PMMA wafer covered by a dry adhesive.

of bonding PMMA to PDMS using surface treatment of PMMA with aminopropyltriethoxysilane (APTES). The bond is irreversible, and any contact with water causes the Si-O-C bond to hydrolyse as reported by Lee et. al. [14]. They treated the PMMA surface with isopropxy modified by bis-trimethoxy-silyl-propyl-amine, which is capable of forming hydrolitically stable bonds between PDMS and modified PMMA.

This paper reports a novel method of bonding between PMMA and PDMS using a dry adhesive film. A hybrid membrane consisting of the adhesive layer and a PDMS layer acts as the pneumatic actuator in a peristaltic micropump. This form of fabrication of the multi-layers not only allows PMMA to be bonded to PDMS, but is also easy to fabricate as well as cost effective. This hybrid membrane worked extremely well, and prevents air from the atuation chambers to leak into the microchannels.

\section{MATERIALS AND METHOD}

PMMA used as the substrate for the micropumps, was purchased from Ying Kwang Acrylic Trading, Singapore. The base polymer and a curing agent of PDMS was purchased from Dow Corning (Sylgard 184). The $50^{1} \mathrm{~m}$ thick dry adhesive was purchased from Adhesive Research (Glen Rock, PA USA). Pressurised nitrogen (SOXAL, Singapore) was used as the pressure source for the membrane tests. Two-way electromagnetic solenoid valves for controlling the pressurised air were purchasedfrom the LEE Co. (Westbrook, CT, USA). Figure 1 depicts the fabrication process of a PMMA/adhesive/PDMS device. The process starts with micromachining of a PMMA substrate. The devices (test membranes and micropumps) were designed using AutoCad 2006 software. The designs are transferred to a commercial CO2 laser system (Universal M-300 Laser Platform, Universal Laser system Inc. Arizona, USA) to engrave the air

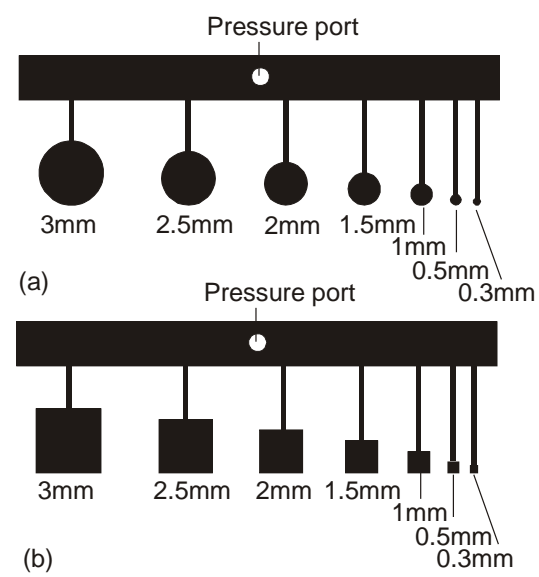

Fig. 1 PDMS membrane designs for the pressure test: (a) circular air chamber, (b) rectangular air chamber.

chambers and the microchannels on the PMMA substrate. The CO2 laser has a wavelength of $10.6 \mu \mathrm{m}$ and a maximum power of $25 \mathrm{~W}$. The maximum speed of the laser beam is $640 \mathrm{~mm} / \mathrm{s}$. The different channel heights can be adjusted by the corresponding laser power and scanning speed. This form of fabrication is fast and cost effective. After laser machining, the PMMA pieces are washed in $70 \%$ ethanol and rinsed with de-ionised (DI) water. Other fabrication techniques such as hot embossing or moulding can be used to form channel structures on the PMMA substrate.

The PMMA substrate was cut into the form of a circular 4-inch wafer. The air chambers are engraved into the PMMA wafer. After cleaning, the dry adhesive film was laminated on the PMMA wafer. The dry acrylic adhesive is is able to bond well to the PMMA surface. In contact with liquids, the adhesive loses its property. Hence, the adhesive film has to be in intimate contact with the PMMA surface. PDMS was prepared, degassed and spin coated onto the PMMA wafer covered by the adhesive ${ }^{-} \mathrm{lm}$. At a rate of $1500 \mathrm{rpm}$ and spinning time of 30 seconds, a PDMS film of $50^{1} \mathrm{~m}$ was formed on the adhesive layer. Thermal curing of the adhesive/PDMS layer took place at $80^{\circ} \mathrm{C}$ for one hour. For the membrane tests, the fabrication process ended here. However, for the fabrication of the peristaltic micropumps, the PMMA wafer with the adhesive/PDMS membrane and the PDMS part with the microchannels underwent oxygen plasma treatment, and were subsequently bonded together. The process results in an irreversible bond.

\section{RESULTS AND DISCUSSIONS}

Figure 2 shows the designs of the membranes used in the pressure test. The test chips contain di ${ }^{\circledR}$ erent air chambers with the shape of squares and circles. The edges or the di- 
ameters range from $300 \mu \mathrm{m}$ to $3000 \mu \mathrm{m}$. The depth of the air chambers in PMMA is $500 \mu \mathrm{m}$. All air chambers share the same pressure port. Investigations of the adhesive/PDMS membrane and the bond strength of both circular and square air chambers were carried out by using a calibrated pressure source. Pressurised nitrogen was first connected to a pressure controller (PPC4 DH Instrument, Fluke Co., USA) which works as the pressure source for the tested membranes. A $45^{\circ}$ mirror (Edmund Optics, Singapore) was placed next to the membrane array to allow imaging both top view and side view of the deformed membrane, Figure 3. The deformation of the membrane was captured by a CCD camera and the corresponding software (Video Savant, IO Industries, Inc., Canada). Images of the deformed membranes were captured, and then analysed by a program written in Matlab (MathWorks, MA, USA). The centre deflection and the radius of curvature of the deformed membrane was measured at different applied pressures. The pressure used for the circular air chambers varies from $2 \mathrm{kPa}$ to $20 \mathrm{kPa}$, whilst for the square air chambers it varies from $2 \mathrm{kPa}$ to $13 \mathrm{kPa}$.

Figure 3 shows the centre deflection and the radius of curvature of circular membranes with different diameters. The results show that the bond quality between PDMS and PMMA is good. No delamination occurs at the maximum pressure of $20 \mathrm{kPa}$. At higher pressures, the membrane would burst while the bond was still intact. The results also indicate that the larger the applied pressure the higher is the centre deflection of the membrane. At the same applied pressure, a larger membrane experiences a larger deflection. The relatively large deflection would allow the adhesive/PDMS membrane to be used as a pneumatic actuator for microvalves and micropumps. The radius of curvature of the deformed membrane decreases with increasing pressure, Fig. 3(b). For the largest membrane with a radius of $3 \mathrm{~mm}$, beyond a critical pressure of about $18 \mathrm{kPa}$ the radius of curvature increases with increasing pressure. This behaviour may be caused by the tear-off effect of the more rigid adhesive layer, making the overall membrane becomes softer. The change in the behaviour of deformation versus pressure can also be observed in the deflection data, Fig. 3(a).

The square membranes were tested up to $13 \mathrm{kPa}$. The reason for the pressure limit is that the deflection of the membrane exceeded the image frame captured by the CCD camera. Similar to the circular membranes, an increasing pressure increases the centre deflection of the membrane and decreases its radius of curvature. Due to the larger membrane area, the deflection for the square membrane was significantly larger than that of a circular. At $20 \mathrm{kPa}$, a 3$\mathrm{mm}$ circular membrane reaches a centre deflection of approximately $2.20 \mathrm{~mm}$. At $13 \mathrm{kPa}$, a $3-\mathrm{mm}$ square membrane reaches a centre deflection of approximately $3.5 \mathrm{~mm}$. Alt-
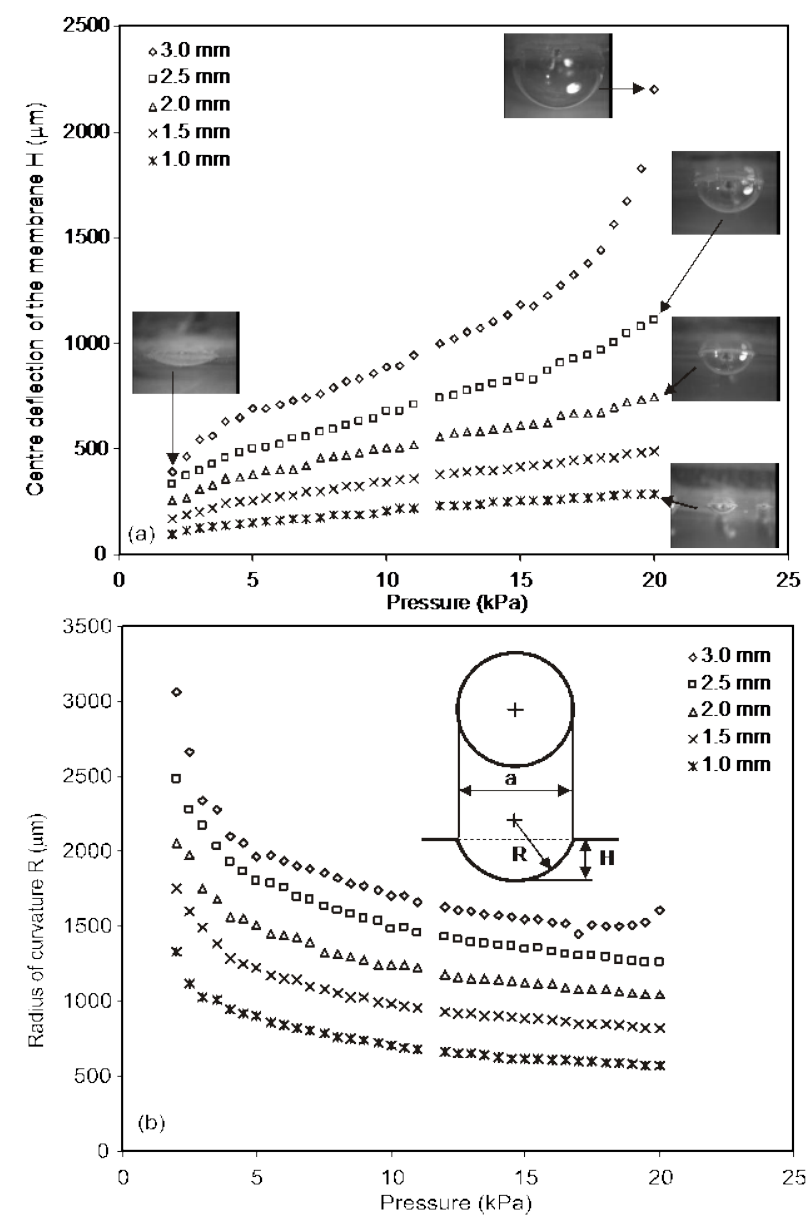

Fig. 3 Geometry of circular membranes at different applied pressures: (a) centre deflection; (b) radius of curvature.

hough the membrane was deformed and took the shape of a balloon, the membrane did not detach from the PMMA substrate. For the same design footprint, a square membrane would allow a larger deflection.

\section{CONCLUSIONS}

We report a novel technique of bonding PMMA to PDMS in a muti-layer polymeric microfluidic device. This technique is based on the concept of laminating a thin layer of double-sided dry adhesive on the PMMA substrate followed by spin coating a layer of pre-cured PDMS mixture. These layers will then undergo thermal curing followed by oxygen plasma to bond irreversibly to another PDMS part. This fabrication technique is suitable for batch processes, and the thickness of the membrane is controllable. Different air chamber sizes and different air pressures were shown to affect the deflection of the membrane. For the same design 

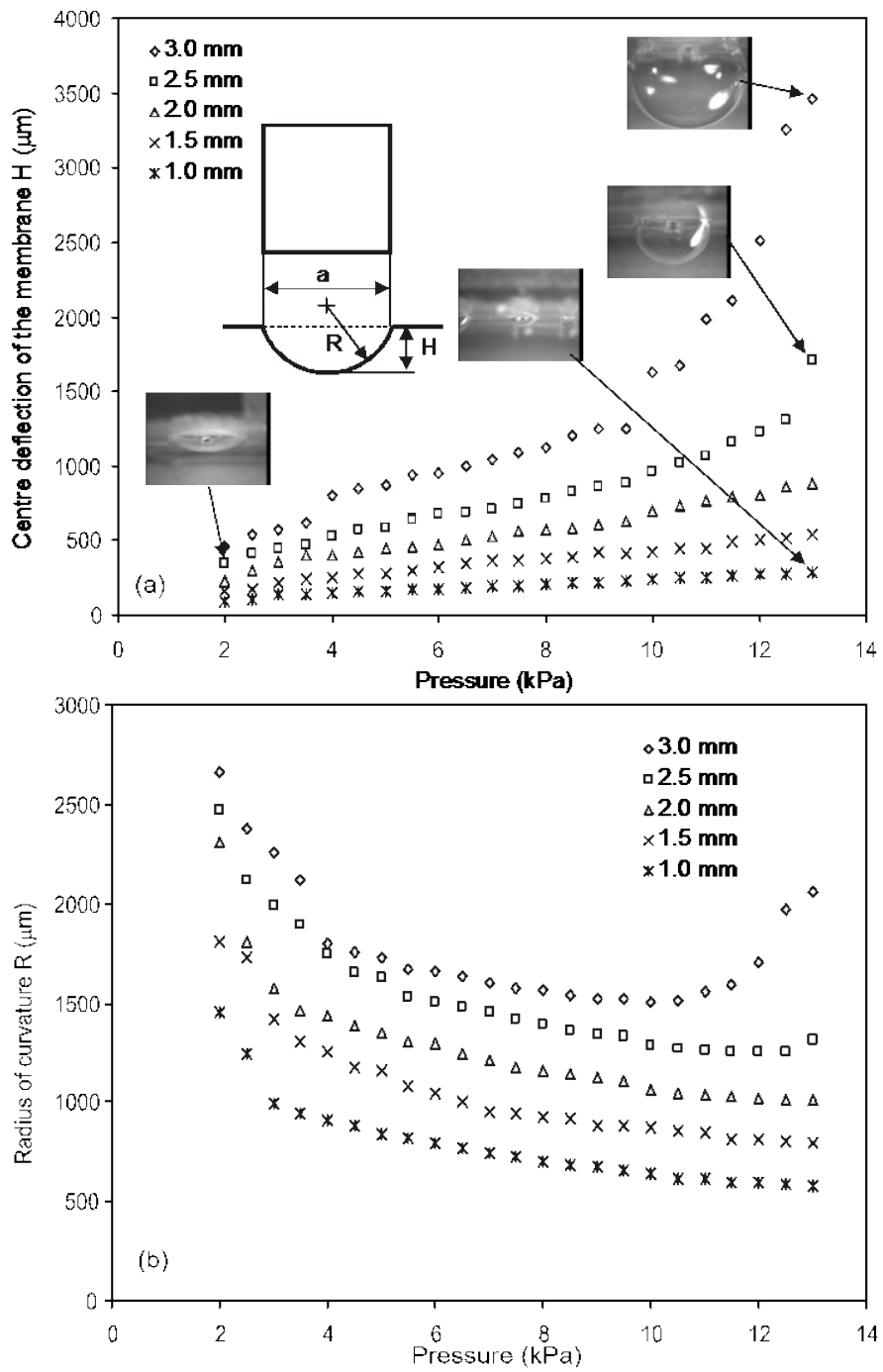

Fig. 4 Geometry of square membranes at different applied pressures: (a) centre deflection; (b) radius of curvature.

foot print, square membranes were able to deflect more than the circular membranes. The square membranes were used in peristaltic micropumps as pneumatic actuators. This novel fabrication technique of the micropumps prevents bubble formation in the microchannel from the infusion of air from the actuation chambers. The layer of adhesive is impermeable to air and adhere well to the PMMA surface. The bond between PMMA and PDMS is strong and reliable as the different layers did not delaminate in the different tests reported in this paper. The micropumps based on the combination of PMMA, dry adhesive and PDMS is powerful, and suitable to work with other components in a more complex LOC platform.

\section{ACKNOWLedgment}

Financial support from Agency for Science, Technology and Research (A*STAR) and Defense Science and Technology Agency (DSTA) of Singapore is gratefully acknowledged (Grant number 08/1/50/19/590).

\section{REFERENCES}

1. Nguyen NT, White RM (2000) Acoustic streaming in micromachined flexural plate wave devices: Numerical simulation and experimental verification. IEEE Transactions on Ultrasonics Ferroelectrics and Frequency Control. 47: 1463-1471

2. Wu ZG, Nguyen NT (2005) Convective-diffusive transport in parallel lamination micromixers. Microfluidics and Nanofluidics. 1: 208-217 DOI: $10.1007 / s 10404-004-0011-x$

3. Wu ZG, Nguyen NT (2005) Rapid mixing using two-phase hydraulic focusing in microchannels. Biomedical Microdevices. 7:13-20 DOI: 10.1007/s10544-005-6167-7

4. Nguyen NT, Huang XY (2005) Thermocapillary effect of a liquid plug in transient temperature fields. Japanese Journal of Applied Physics Part 1 - Regular Papers Brief Communications \& Review Papers. 44: 1139-1142 DOI: 10.1143/JJAP.44.1139

5. Nguyen NT, Ng KM, Huang XY (2006) Manipulation of ferrofluid droplets using planar coils. Applied Physics Letters. 89: 052509 DOI: 10.1063/1.2335403

6. Ting TH, Yap YF, Nguyen NT, et al. (2006) Thermally mediated breakup of drops in microchannels. Applied Physics Letters. 89: 234101 DOI: $10.1063 / 1.2400200$

7. Unger MA, Chou HP, Thorsen T et al. (2000) Monolithic microfabricated valvesand pumps by multilayer soft lithography. Science 288:113-116

8. Zhang W, Lin S, Wang C et al. (2009) PMMA/PDMS valves and pumps for disposable microfluidics. Lap Chip 9:3088-3094 DOI: 10.1039/b907254c

9. Kim JH, Lau KT, Shepherd R et al. (2008) Performance characteristics of a polypyrrole modified polydimethylsiloxane (PDMS) membrane based microfluidic pump. Sensors and Actuators A 148: 239244 DOI: 10.1016/j.sna.2008.07.029

10. Abgrall P, Nguyen NT (2008) Nanofluidic devices and their applications. Analytical Chemistry. 80:2326-2341 DOI: 10.1021/ac702296u

11. Abgrall P, Low LN, Nguyen NT (2007) Fabrication of planar nanofluidic channels in a thermoplastic by hot-embossing and thermal bonding. Lab on a Chip. 7:520-522 DOI: 10.1039/b616134k

12. Toh AGG, Ng SH and Wang ZP (2009) Fabrication and testing of embedded microvalves within PMMA microfluidic devices. Microsyst. Techno. 15:1335-1342 DOI: 10.1007/s00542-009-0821-6

13. Vlachopoulou ME, Tserepi A, Pavli P et al. (2009) A low temperature surface modification assisted method for bonding plastic substrates. J. Micromech. Microeng. 19:015007 DOI: 10.1088/09601317/19/1/015007

14. Lee KS and Ram RJ (2009) Plastic-PDMS bonding for high pressure hydrolytically stable active microfluidics. Lab Chip 9:1618-1624 DOI: $10.1039 / b 820924 c$

Author: Nam-Trung Nguyen

Institute: Nanyang Technological University

Street: 50 Nanyang Avenue

City: $\quad$ Singapore

Country: Singapore

Email: mntnguyen@ntu.edu.sg 